\title{
Stimulus versus response probability effects in choice reaction time ${ }^{1}$
}

\author{
IRVING BIEDERMAN ${ }^{2}$ AND RICHARD A. ZACHARY \\ STATE UNIVERSITY OF NEW YORK AT BUFFALO
}

\begin{abstract}
The effects of stimulus and response probability on choice reaction time (RT) were independently investigated. In different conditions, two sets of three stimuli of probabilities of $.1, .3, .6$ and .3 , $.3, .4$ were assigned to two responses-two of the stimuli assigned to one response. All combinations of $S-R$ assignments were studied, yielding response probabilities of $.1-9, .3-7$, and .4-6. With response probability held constant, variations in stimulus probability led to consistent and substantial effects on both $R T$ and error rates. Response-probability effects were confined to error rates, and repetition effects were negligible. The results support the assumption of recent models of choice behavior that adjustments are made to the absolute (rather than just to the ordinal) values of stimulus probability.
\end{abstract}

Of fundamental importance in man's capacity for processing information is his ability to adjust to variations in stimulus probability. The regularity of the relationship between stimulus probability and choice reaction time (RT) is truly striking: negative correlations in the high $.90 \mathrm{~s}$ are commonly obtained (Hyman, 1953; Fitts, Peterson, \& Wolpe, 1963). The mechanism(s) responsible for this relationship are thus considered to be central to most theoretical accounts of human information-processing behavior (Smith, 1968).

Given the theoretical importance of stimulus probability, it is surprising to note that the manner in which this variable influences processes intervening betiveen stimulus and response has only recer, tly received empirical study (Bertelson \& Tisseyre, 1966; LaBerge \& Tweedy, 1964). The present experiment was designed to investigate several interrelated questions concerning the relationship between stimulus probability and RT. The central question is: Do variations of stimulus probability primarily affect processes involved in perceiving (or in deciding) which stimulus has been presented, or do they primarily affect processes involved in the selection and/or execution of responses? It should be recognized that the two alternatives of this issue are not mutually exclusive, and that it is obviously possible for a variable to influence both. Thus, the issue may be rephrased as an assessment of the relative magnitudes of the stimulus and response effects under stated experimental conditions.

Early experiments manipulating stimulus probability (Hick, 1952; Hyman, 1953) confounded stimulus and response probability by assigning stimulus to response in a $1: 1$ fashion. Consequently, it is uncertain whether the shorter RTs to the more probable stimulus resulted from the stimulus being more probable or because the response assigned to that stimulus was more probable. LaBerge and Tweedy (1964) controlled for response effects by using a many:one stimulus-to-response assignment, where the different stimul assigned to the same response had different probabilities of occurrence. They found that shorter RTs occur to the more probable stimulus, which is evidence for a stimulus effect. A similar result was reported by Bertelson and Tisseyre (1966).

The presence of a response effect can be ascertained if the same set of stimulus probabilities are reassigned to the same set of responses but with a different many:one mapping so that the response probabilities differ from the previous condition. An example illustrates this logic. Consider three stimuli with probabilities of $.1, .3$, and .6 and two responses, $\mathrm{Ra}$ and $\mathrm{Rb}$. In one condition, the .1 and .3 probability stimuli could be assigned to $\mathrm{Ra}$ and the .6 probability stimulus to $\mathrm{Rb}$. If $\mathrm{RT}$ s to the .3 probability stimulus are shorter than those to the .1 probability stimulus, then a stimulus effect is evidenced since the response (of probability .4) is held constant. In another condition, the .1 and .6 probability stimuli could be assigned to $\mathrm{Ra}$ and the .3 probability stimulus to $\mathrm{Rb}$. A response effect is evidenced if $R T$ s to the .1 probability stimulus are shorter in this condition than they were in the first. In this comparison, the response probability is varied from .4 to .7 , while the stimulus probability is held constant at .1. Bertelson and Tisseyre (1966) found no evidence for a response effect when stimulus probability was held constant at .15 and response probability varied from .30 to .70 .

The present experiment was also designed to determine whether stimulus probability effects on $R T$ reflect the absolute or the ordinal magnitudes of these probabilities. LaBerge and Tweedy (1964) used a many:one stimulus-response mapping with only a single set of probabilities, .1, .4, and .5, with the .1 and
.5 probability stimuli assigned to one response and the .4 probability stimulus assigned to the other. While a stimulus effect was found in that the RTs that occurred to the .5 probability stimulus were shorter than those that occurred to the .1 probability stimulus, it is not clear whether this effect is attributable to the absolute probabilities (.5 vs .1) or solely to ordinal differences in probability.

This issue is relevant to the way in which different theorists have conceptualized the nature of S's adjustment to probabilities. Thus, stimulus-sampling and decision-making models (Fitts, 1965; Fitts, Peterson, \& Wolpe, 1963; Stone, 1960) have posited a central "decision maker" that processes the information on the basis of the absolute values of the a priori stimulus probabilities. In contrast, some of the sequential-search models (Hick, 1951; Welford, 1959) assert that stimulus probability effects simply reflect the internal, fixed sequence in which the stimulus representations are scanned. Consequently, only the ordinal values of the probabilities would be relevant in determining the search sequence. Sequential-search models can be modified so that they are compatible with adjustments to absolute probabilities by assuming that the scanning sequence changes from trial to trial, and that the probability of stimuli being scanned in a given order is related to the absolute values of the stimulus probabilities. However, such an assumption represents a major change in the character of those search models that attempted to account for probability effects via a fixed, sequential scan in a manner analogous to an efficient coding sequence derivable from information theory (Hick, 1952).

One way of determining whether stimulus probability functions absolutely or ordinally is to use two sets of different probabilities in which the sum of the probabilities of two of the stimuli in one set equals the sum of the probabilities of two of the stimuli in the other set. The present experiment used two distributions of stimulus probabilities: $.1, .3, .6$ and .3 , .3, .4. All three combinations of assigning the three stimuli to two responses were studied. There were, therefore, two conditions in which the response probability was .7. In one condition, it was comprised of two stimuli with probabilities 


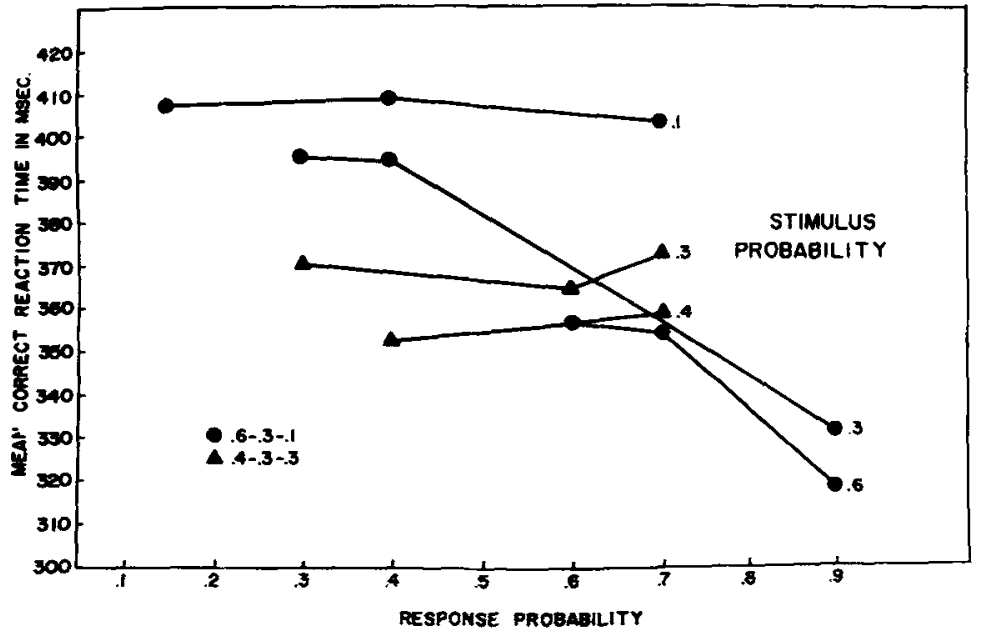

of .1 and .6; in the other condition, the probabilities were .3 and .4 . If the difference in RTs in the former condition is greater than in the latter, it is evidence for the role of the absolute magnitudes in the production of the stimulus-probability effect.

Finally, the effects of these variables on the accuracy of S's response were also studied. This is particularly important in an RT task since $S$ possesses a most impressive capacity for trading off speed for accuracy (Fitts, 1966).

\section{Subjects}

\section{METHOD}

Twenty-four male students, with normal color vision, from the State University of New York at Buffalo, participated in the experiment as part of their introductory course requirement in psychology.

\section{Stimuli}

Two sets of three stimuli were used. The first set of stimuli were colored circles of radius $14.1 \mathrm{~mm}$. The three colors were red, green, and blue Colormat papers $(63,140$, 163) that were approximately matched with Munsell standards so that they were equal on saturation and value. The second set consisted of three shapes: square, triangle, and circle. All three shapes were blue and were of equal area. The square was $25 \mathrm{~mm}$ on a side, the triangle was $37.9 \mathrm{~mm}$ on a side, and the circle had a radius of $14.1 \mathrm{~mm}$. All stimuli were mounted on a white paper background, centered on $8.3 \times 10.2 \mathrm{~cm}$ glass slides.

\section{Apparatus}

Stimuli were presented in a Polymetric Tachistoscope (Model V0959T), modified with a rotatable carrier that held three slides. The pre- and postexposure fields, as well as the background field, were a uniform white. S's index fingers rested on plastic switches that activated a microswitch. RTs were measured by a Lafayette Instrument Co. Standard Electric Timer to the nearest $1 / 100$ of a second. A set of lights on the E's panel indicated S's response. The sequence of ready signal, presentation, and reset was controlled by a Lafayette Instrument Co. VIII Bank Timer (Model 1431A). Stimulus presentation and response recording were done by $\mathrm{E}$.

\section{Procedure}

The $S$ was seated and the $T$-scope adjusted to his line of sight. He was fully instructed as to the nature of the stimuli, their probabilities, and their assignments to fingers, and a card displaying this information was placed by the response panel and remained there while $S$ performed. The three stimuli were then shown in the tachistoscope, and instructions were given. $\mathrm{S}$ was told to respond as fast and as accurately as possible.

The experimental session consisted of three blocks of 100 trials each. Successive pairs of stimuli were represented in each sequence in proportion to the product of their probabilities. The interstimulus interval was $10 \mathrm{sec}$, with a warning buzzer of $.5-\mathrm{sec}$ duration, sounding $1 \mathrm{sec}$ before the stimulus presentation of $.5 \mathrm{sec}$. $\mathrm{S}$ was verbally provided error and RT feedback (to the nearest $.01 \mathrm{sec}$ ) after each response. Ten practice trials were given before each of the trial blocks. Approximately $2 \mathrm{~min}$ were required between blocks for instructions for the next task.

\section{Design}

The Ss were equally divided into groups that differed in the distribution of probabilities over the set of three stimuli. For one group (.1-.3-.6 set), the stimuli had probabilities of $.1, .3$, and .6. Each $S$ received all three possible combinations of assigning the three stimuli to two responses. Therefore, the conditions for
Fig. 1. Mean correct RT as a function of stimulus and response probability.

this set were (arbitrarily designating the two responses as $R a$ and $R b$ ): .1 and .3 assigned to $\mathrm{Ra}$ and .6 to $\mathrm{Rb}, .1$ and .6 to $\mathrm{Ra}$ and .3 to $\mathrm{Rb}$, and .3 and .6 to $\mathrm{Ra}$ and .1 to $\mathrm{Rb}$. $\mathrm{Ra}$, consequently, had probabilities of $.3, .4$, and .9 , while $\mathrm{Rb}$ had the complementary probabilities. Three conditions of another set (.3-.3-.4) were formed in a manner analogous to that of the .1-.3-.6 set. Two of these conditions were indistinguishable with respect to the probabilities of their S-R assignments (they had a .3 and .4 probability stimulus assigned to one response and $a .3$ probability stimulus assigned to the other). Since a within-Ss design was used, with each $S$ getting all three conditions and the order counterbalanced between Ss, repeating the probability structure of a condition was necessary to equate practice effects between the two stimulus sets.

Half the Ss within each probability set were presented with stimuli differing only in color, while the other half were presented with stimuli differing only in shape. The assignment of a probability to a stimulus did not change over conditions for a given $S$. However, the assignment of the three probability levels to each stimulus was balanced over all Ss within a set. Also controlled was the assignment of probabilities to the preferred vs the nonpreferred hand.

\section{RESULTS AND DISCUSSION}

The variables of stimulus dimension (shape vs color) and hand preference had negligible effects and, consequently, will be ignored. Effects attributable to learning were also slight and will not be discussed further.

The results provide strong support for a stimulus effect. Figure 1 shows mean correct RTs as a function of both stimulus and response probability. The main effect of stimulus probability was highly significant in the $.6-.3-.1$ set $[F(2,20)=20.70, p<.001]$ and just short of significance in the $.4-.3-.3$ set $[\mathrm{F}(2,20)=3.55, .10<\mathrm{p}<.05]$.

In the four conditions where two stimuli of unequal probability were assigned to the same response (.1-.3, .1-.6, .3-.6, and .3-.4), shorter RTs were associated with the more probable stimulus. That this is not simply an ordinal effect is evidenced by the greater difference in RTs between .1-.6 than between .3-.4 (the response probability being .7 in both cases). It is possible to formulate a search model based solely on the ordinal values of stimulus probability that is compatible with the 


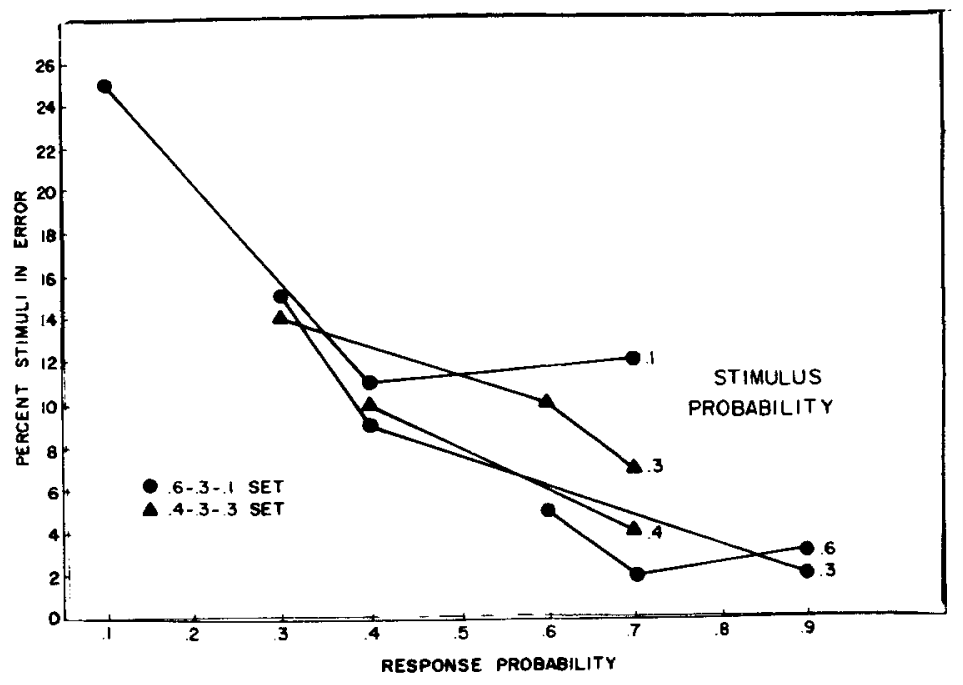

obtained result of a greater RT difference between the .1-.6 stimuli than with the .3-.4 stimuli. However, the implications of this model are inconsistent with other aspects of the results, which will now be examined. In the .6-.3-.1 set, the ordinal search model would hold that the .6 probability stimulus would be examined first, the .3 stimulus second, and the .1 stimulus last. In the $.4-.3-.3$ set, the .4 probability stimulus would be examined first, then on half the trials the .3 stimulus that is assigned in conjunction with the .4 stimulus, and last, the .3 stimulus that is assigned in 1:1 fashion. On half the trials, the order of examination of the last two stimuli would be reversed. (An equal sequential priority is supported by the data in that the .3 probability stimulus assigned by itself had approximately the same RT as the .3 probability assigned in conjunction with the .4 probability stimulus.) Thus, the .3 stimulus would always be examined second in the $.6-3-.1$ set and second on half the trials and third on the other half of the trials in the $.4-.3-.3$ set. Consequently,

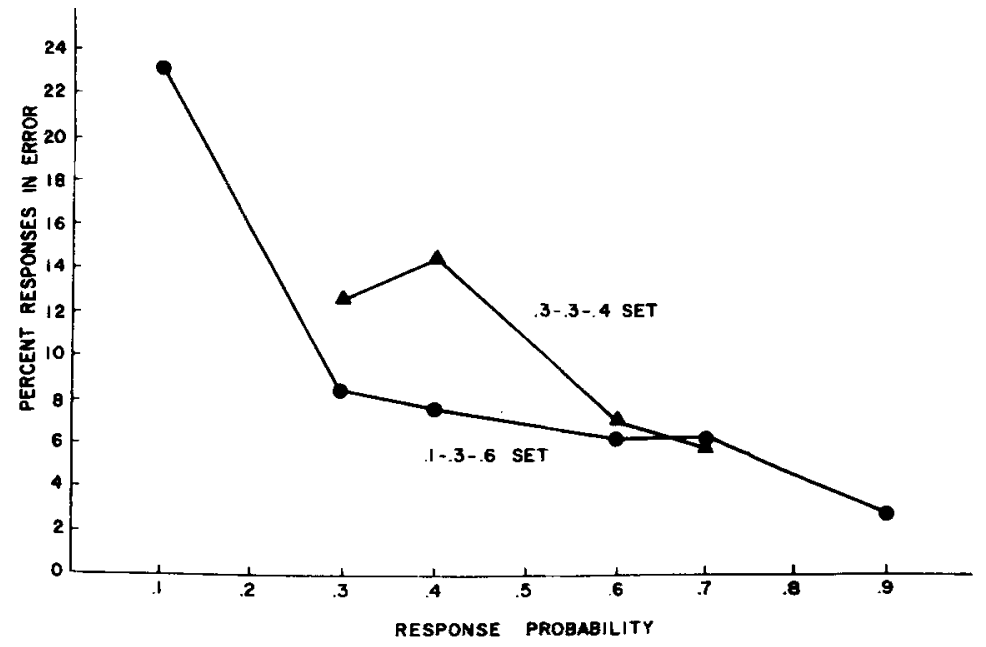

Fig. 2. Mean per cent errors as a function of stimulus and response probability.

the two .3 probability stimuli are assigned to the same response. Together, therefore, they sum to a higher response probability (.6) than does the .4 probability stimulus. Yet, the RT to the .4 probability stimulus is $352 \mathrm{msec}$, which is still shorter than the RTs to each of the .3 probability stimuli, which average $364 \mathrm{msec}$.

The evidence for a response effect (holding stimulus probability constant) is somewhat more equivocal. In Fig. 1, response effects are shown as decreases in the RT functions for individual stimuli as response probability increases. The only substantial decrease in RT attributable to response probability occurs with the .9 probability response in the $.6-.3-.1$ set and accounts for a significant Probability by Condition interaction for that set $[F(4,40)=5.30, p<.01]$. Between the range of response probabilities .1 to .7 , the RT functions are flat and the $F$ ratio for the Probability by Conditions interaction in the $.3-.3-.4$ set is less than 1.00 .

Since, in these tasks, it is possible to trade speed for accuracy, the above results for RTs must be evaluated in the context of the error data. Figure 2 shows that there was a consistent relationship between both stimulus and response probability and mean per cent errors. In general, as stimulus or response probability increased, the error rates decreased. However, in order to evaluate a speed-for-accuracy tradeoff, errors should also be expressed in terms of the proportion of times that a response, when made, was in error (Fitts, Peterson, \& Wolpe, 1963). These data are shown in Fig. 3. There is a general decrease in error rates as response probability is increased. Given that erroneous RTs are shorter than correct RTs, a relatively greater proportion of the low-probability responses would have benefited from a speed-accuracy tradeoff. Consequently, response-probability effects are underestimated.

Since the stimulus sequences were generated independently, the probability of a stimulus was confounded with the probability of its repetition. In some experiments, RTs to stimuli that are repeated from the previous trial tend to be faster than RTs to nonrepetitions (Kornblum, 1969). It is therefore necessary

Fig. 3. The percentage of trials that a response, when made, was in error as a function of the a priori probability of that response. 
to determine the extent to which sequential effects can account for the relations between stimulus probability and RT reported above.

Sequential effects were examined through a categorization of correct RTs on Trial $\mathbf{n}$ as identical (same stimulus as on Trial $\mathbf{n}-1$ ), equivalent (different stimulus but same response as on Trial $n-1$ ), or different (different stimulus and different response as on Trial $n-1)$. Trials following an error were not included in this analysis. In the .6-.3-.1 condition, RTs for identical, equivalent, and different trials were 376,376 , and $379 \mathrm{msec}$, respectively. In the .4-3-.3 condition, these RTs were 368,368 , and $358 \mathrm{msec}$, respectively. The overall sequential effect was, therefore, negligible. Separate plots for identical, equivalent, and different RTs show only small and unsystematic departures from the overall results shown in Fig. 1. This study thus joins LaBerge and Tweedy's (1964) study in demonstrating stimulus-probability effects unconfounded by sequential effects. Perhaps it was the relatively long ISIs in both LaBerge and Tweedy's experiment and the present one, 3 and $10 \mathrm{sec}$, respectively, or perhaps it was the use of a two-choice response that accounts for the absence of sequential effects. Further research is needed to determine the conditions under which such effects are manifested.

It is interesting to consider the implications of stimulus-probability effects on the memory-scan paradigm so ingeniously explored by Sternberg (1967). Sternberg controlled for stimulus and response entropy and response probability when he varied the number of iterns in the positive set. However, the number of items in the positive set was confounded with their probability of occurrence-items in set sizes of 1,2 , and 4 had probabilities of occurrence of $4 / 15,2 / 15$, and $1 / 15$, respectively. On the basis of equal slopes for the "YES" and "NO" responses, Stermberg postulated an exhaustive sequential scan of the items in the positive set. It can be argued that this scanning is related only to number of iterns and not to their probability from the absence of a relation between RT and the probability of items in the negative set. Since it was assumed that $S$ s were scanning only the positive set, the absence of such a relation would be expected whether the number of items in the positive set or their probability was the effective variable.

A more critical test would involve varying the probability of items within a given positive set. For example, in
Sternberg's (1967) experiment, when there were two items in the positive set, their probabilities were both $2 / 15$. If one item had a probability of $1 / 15$ and the other, $3 / 15$, then exhaustive sequential scanning would not predict faster RTs to the more probable item.

Insofar as a number of studies have now documented stimulus-probability effects, Dillon's (1966) failure to find such effects warrants some scrutiny. The major methodological difference between Dillon's procedure and other studies using many-to-one mappings and variations in stimulus probability was Dillon's use of a response-demand signal. Thus, Dillon's Ss did not respond on every trial but, instead, responded only if a tone occurred after the stimulus (one of eight taped letters) was presented. It seems reasonable to assume that stimulus processing would largely be completed during the relatively long interval $(0.5 \mathrm{sec})$ between the stimulus and the response-demand signal; LaBerge and Tweedy (1964) and Bertelson and Tisseyre (1965), as well as the present study, report RTs in the 350- to 500-msec range. Consequently, it is not at all surprising to find an absence of stimulus-probability effects. Indeed, it would not be at all surprising to find an absence of response-probability effects under Dillon's conditions. In fact, examination of Dillon's data reveals that response-probability differences as large as .80 vs .20 and .85 vs .15 fail to produce RT effects. Further supporting this interpretation of processing completion during the response-demand interval are the extremely short latencies of Dillon's Ss-often under $200 \mathrm{msec}$ and some as low as $130 \mathrm{msec}$. It is thus likely that Dillon was obtaining simple $\mathrm{C}$ reactions to the auditory response-demand signal and what probability effects he did obtain were largely those for the go/no-go choice. All in all, Dillon's suggestion that ". . . models of DRT performance should stress response-selection, rather than stimulus-identification, factors in decision processing [p. 321]" would appear to be, at best, premature.

The results of the present experiment are consistent with the conception of the human having the capacity to make absolute adjustments to stimulus probability. However, additional research is required to determine more precisely where, in the sequence of events prior to response selection, these adjustments occur. One strategy for this task is to study the interaction between the magnitude of the stimulus-probability effect and variables whose effects are specific to given stages (e.g., stimulus discriminability).

It should be also noted that, in the present experiment, the stimulus-probability effect was substantially greater than the response-probability effect. Further study is needed to define the conditions governing the magnitudes of these effects.

\section{REFERENCES}

BLACKMAN, A. R. Stimulus and response probability and choice reaction time. Paper presented at the meeting of the Psychonomic Society, St. Louis, November 1968.

BERTELSON, P., \& TISSEYRE, F. Choice reaction time as a function of stimulus versus response relative frequency of occurrence. Nature, 1966, 212, 1069-1070.

DILLON, P. J. Stimulus versus response decisions as determinants of the relative frequency effect in disjunctive reaction time performance. Journal of Experimental Psychology, 1966, 71, 321-330.

FITTS, P. M. Cognitive aspects of information processing: III. Set for speed vs. accuracy. Journal of Experimental Psychology, 1966, 71, 321-330.

FITTS, P. M., PETERSON, J. R., \& WOLPE, G. Cognitive aspects of information processing: II. Adjustments to stimulus redundancy. Journal of Experimental Psychology, 1963, $65,423-432$.

HICK, W. E. On the rate of gain of information. Quarterly Journal of Experimental Psychology, 1952, 4, 11-26.

HYMAN, $R$. Stimulus information as a determinant of reaction time. Journal of Experimental Psychology, 1965, 69, 511-514.

KORNBLUM, S. Sequential determinants of information processing in serial and discrete choice reaction time. Psychological Review, 1969, 76, 113-131.

LaBERGE, D., \& TWEEDY, J. R. Presentation probability and choice time. Journal of Experimental Psychology, 1964, 68, 477-481.

SMITH, E. E. Choice reaction time: An analysis of the major theoretical positions. Psychological Bulletin, 1968, 69, 77-110.

STERNBERG, S. Two operations in character recognition: Some evidence from reaction-time measurements. Perception \& Psychophysics, $1967,2,45-53$.

STONE, M. Models for choice-reaction time. Psychometrika, 1960, 25, 251-260.

WELFORD, A. T. The measurement of sensory-motor performance: Survey and reappraisal of twelve years" progress. Ergonomics, 1960, 3, 189-229.

\section{NOTES}

1. The assistance of Stephen F. Checkosky, Joseph S. Dumas, and Erwin M. Segal is gratefully acknowledged. A. R. Blackman (1968), working independently of the authors, recently reported an experimental investigation and results that are, in part, similar to those of the present experiment.

2. Address: Department of Psychology, State University of New York at Buffalo, Buffalo, New York 14226.

(Accepted for publication June 17, 1969.) 